\title{
ANTECEDENTES DA INTENÇÃO DE RECOMPRA DE UMA MARCA DE SMARTPHONES ${ }^{l}$
}

\author{
Gabriel Sperandio Milan ${ }^{2}$ \\ Vinicius Zanchet de Lima ${ }^{3}$ \\ Luciene Eberle ${ }^{4}$ \\ Deonir De Toni ${ }^{5}$
}

http://dx.doi.org/10.1590/1413-2311.165.61873

\begin{abstract}
RESUMO
A intenção de recompra possui grande importância no contexto acadêmico e empresarial e, por este motivo, diversos pesquisadores salientam que há lacunas para aprofundar o conhecimento sobre os seus construtos antecedentes. Compreender o fenômeno que leva o consumidor a ter a intenção de recompra de certo produto e/ou serviço de uma marca é um aspecto chave para o sucesso das empresas. Diante disso, o presente estudo analisou a influência do valor percebido, do comprometimento afetivo, do comprometimento normativo e da propaganda boca a boca positiva sobre a intenção de recompra de smartphones de uma determinada marca. Para tanto, foi realizada uma pesquisa quantitativa, por meio da aplicação de uma survey. A análise dos resultados foi feita através de estatísticas multivariadas, utilizando-se a técnica da Modelagem de Equações Estruturais para observar e analisar as variáveis que compõem o modelo teórico proposto, através da realização do teste das hipóteses levantadas. A amostra foi composta por 411 consumidores de uma das marcas líderes de mercado de smartphones. Os resultados confirmaram as sete hipóteses testadas, evidenciando o valor percebido, o comprometimento afetivo, o comprometimento normativo, a propaganda boca a boca positiva como antecedentes da intenção de recompra de um smartphone de uma marca específica. Assim, os resultados do presente estudo possibilitam refletir sobre algumas contribuições relevantes do modelo teórico testado em relação à intenção de recompra.
\end{abstract}

Palavras-chave: Valor Percebido. Comprometimento Afetivo. Comprometimento Normativo. Intenção de Recompra. Modelagem de Equações Estruturais.

\section{THE ANTECEDENTS OF THE INTENTION TO REPURCHASE \\ A BRAND OF SMARTPHONES}

\footnotetext{
${ }^{1}$ Recebido em 27/01/2016; aprovado em 02/05/2017.

${ }^{2}$ Universidade de Caxias do Sul - gsmilan@ucs.br.

${ }^{3}$ Universidade de Caxias do Sul - vinizanchet@gmail.com.

${ }^{4}$ Universidade de Caxias do Sul - leberle@ucs.br.

${ }^{5}$ Universidade de Caxias do Sul-DToni2@ucs.br.
} 


\begin{abstract}
The repurchase intention has great importance in the academic and business environments and, for this reason, many researchers point out that the gaps related to the understanding of previous constructs should be solved by the promotion of studies and, as a consequence, the increase of knowledge. Understanding the phenomenon that makes the consumer have the intention to repurchase a specific product and / or service of a brand is a key aspect to the success of companies. Thus, the present study examined the influence of: perceived value, affective commitment, normative commitment and positive mouth propaganda in relation to the intention to repurchase a smartphone of a particular brand. To this end, a quantitative research was conducted through the application of a survey. The analysis was performed using multivariate statistics, using the technique of structural equation modeling to observe and analyze the variables that make up the proposed theoretical model, through the testing of hypotheses. The sample consisted of 411 consumers of one of the leading brands of smartphones. The results confirmed the seven hypotheses tested, indicating the perceived value, the affective commitment, normative commitment and positive mouth propaganda as antecedents of the intention to repurchase a smartphone of a specific brand. The results of this study make it possible to reflect on some outstanding contributions tested through the theoretical model regarding the repurchase intention.
\end{abstract}

Keywords: Perceived Value. Affective Commitment. Normative Commitment. Repurchase Intentions. Structural Equation Modeling.

\title{
LOS ANTECEDENTES DE LA INTENCIÓN DE RECOMPRA \\ DE UNA MARCA DE SMARTPHONES
}

\begin{abstract}
RESUMEN
La intención de recompra tiene gran importancia en el ámbito académico y empresarial y, por esta razón, muchos investigadores señalan que existen lagunas y es necesario aumentar el conocimiento acerca de sus construcciones antecedentes. Comprender el fenómeno que lleva al consumidor a tener la intención de recompra de determinado producto y / o servicio de una marca es un aspecto clave para el éxito empresarial. Por lo tanto, el presente estudio examinó la influencia del valor percibido, del compromiso afectivo, del compromiso normativo y de la propaganda boca a boca positiva, en la intención de recompra de una marca de smartphones en particular. Con este fin, un estudio cuantitativo se llevó a cabo mediante la aplicación de una survey. El análisis se realizó utilizando estadísticas multivariantes, usando la técnica de modelos de ecuaciones estructurales para observar y analizar las variables que componen el modelo teórico propuesto, a través de la prueba de hipótesis. La muestra consistió en 411 consumidores de una de las principales marcas de smartphones. Los resultados confirmaron las siete hipótesis probadas, evidenciando el valor percibido, el compromiso afectivo, el compromiso normativo y la propaganda boca a boca positiva como antecedentes de la intención de recompra de un producto de una marca específica. Los resultados de este estudio permiten reflexionar sobre algunas contribuciones importantes del modelo teórico examinado en relación con la intención de recompra.
\end{abstract}

Palabras-clave: Valor Percibido. Compromiso Afectivo. Compromiso Normativo. Intención de Recompra. Ecuaciones Estructurales. 


\section{INTRODUÇÃO}

Atualmente, para que as empresas alcancem o sucesso nos negócios torna-se primordial compreender os processos de tomada de decisão dos clientes para predizer as suas intenções e os seus comportamentos de compras futuras. Neste sentido, a intenção de recompra dos clientes tem sido o objetivo de alguns estudos na área de marketing, principalmente associados às teorias do comportamento do consumidor (HAN; KIM, 2010). Cabe ressaltar que a intenção de recompra é o processo de um indivíduo (cliente ou consumidor) em adquirir produtos e/ou serviços de uma mesma empresa ou marca (HELLIER et al., 2003), sendo considerada como um fator importante nas atitudes e nos comportamentos dos clientes, e que, muitas vezes são influenciados por normas subjetivas, que são formadas pelas crenças e pelos aspectos intrínsecos à interação social (LI; HONG, 2013).

Neste horizonte, a literatura acerca da intenção de recompra apresenta desafios para a sua maior compreensão e consolidação (HE; SOUG, 2009), sendo que há vários estudos voltados ao aprofundamento da compreensão de como estimular o cliente e/ou o consumidor a desenvolver a intenção de recompra de uma marca e de um produto e/ou serviço, junto a uma empresa ao longo do tempo (OLARU; PURCHASE; PETERSON, 2008; HE; SOUG, 2009; LEE; EZE; NDUBISI, 2011; VAERENBERGH; LARIVIÈRE; VERMEIR, 2012; LIN; LEKHAWIPAT, 2014; HSU et al., 2014).

Diante disso, é premente a necessidade da busca do conhecimento sobre as relações entre os construtos antecedentes (ou determinantes) da intenção de recompra (TSAI; HUANG, 2007; HE; SONG, 2009; HAN; RYU, 2012). Por conseguinte, o valor percebido é reconhecido como um antecedente da intenção de recompra (MOLINER et al., 2007), devido ao fato que os clientes podem recomprar um mesmo produto e/ou serviço de uma mesma empresa, ou marca, se perceberem que estão recebendo maior valor do que possivelmente a oferta do concorrente (WU et al., 2012).

Avançando na discussão, o comprometimento também é reconhecido como um importante antecedente da intenção de recompra (JONES et al., 2007; HAN; RYU, 2012; ERCIS et al., 2012), sendo considerado relevante para se entender o comportamento do comprador (PALMATIER et al., 2006), pois se refere a um sinal explícito ou implícito da continuidade da relação entre os parceiros de troca em uma perspectiva de longo prazo (MORGAN; HUNT, 1994). Além disso, Pritchard, Havitz e Howard (1999) salientam que o comprometimento pode ser compreendido como uma forte resistência à mudança, e que resulta em comportamentos de repetição de negócios (ISAID; FAISAL, 2015). Contudo, é 
importante salientar que o comprometimento é conceituado por diversos pesquisadores por meio de uma abordagem multidimensional (FULLERTON, 2011; ALVES; TERRES; SANTOS, 2013), ou seja, formado por dimensões. No caso específico do presente estudo, foram consideradas duas dimensões, ou seja, as dimensões do comprometimento afetivo e do comprometimento normativo.

Outro construto que possui uma relação sobre a intenção de recompra é a propaganda boca a boca positiva (LIU; LEE, 2016; KITAPCI; AKDOGAN; DORTYOL, 2014). Trata-se de uma comunicação verbal, informal e pessoal entre um comunicador e um receptor, sendo entendida como uma recomendação ou indicação a terceiros de uma empresa, de uma marca ou de um produto e/ou um serviço (HARRISON-WALKER, 2001; ENNEW; BANERJEE; LI, 2000). É imperioso salientar que a propaganda boca a boca é um componente relevante no contexto do marketing, pois influencia os processos de tomada de decisão de compra, além de ser eficaz para a continuidade dos relacionamentos ao longo do tempo (DINH; MAI, 2016).

Em se tratando do contexto de pesquisa, a expansão do mercado de telefonia móvel, principalmente dos smartphones, têm despertado o interesse de diversos pesquisadores (REVELS; TOJIB; TSARENKO, 2010; SHIN et al., 2011; ISAID; FAISAL, 2015). Nos últimos anos, a compra de smartphones tem sido acelerada pelo avanço das tecnologias, marcas, modelos, níveis de preço e tendências, porém com um ciclo de vida breve (WANG; XIANG; FESENMAIER, 2014). Por isso, o desenvolvimento de pesquisas sobre telefonia móvel e principalmente sobre smartphones são oportunas para as empresas entenderem quais estratégias e ações mercadológicas devem ser adotadas neste mercado para que lhe alavanquem negócios no futuro com os consumidores existentes e os consumidores potenciais (TING et al., 2011).

Assim sendo, a questão central de pesquisa que norteou o desenvolvimento deste estudo foi a seguinte: Qual a influência do valor percebido, do comprometimento afetivo, do comprometimento normativo e da propaganda boca a boca positiva sobre a intenção de recompra de smartphones por parte do consumidor? Em decorrência desta questão central de pesquisa, foi delineado o objetivo central da pesquisa, que é o de analisar o impacto do valor percebido, do comprometimento afetivo, do comprometimento normativo e da propaganda boca a boca positiva sobre a intenção de recompra de smartphones de uma determinada marca (a segunda marca em vendas no mercado mundial). 


\section{REFERENCIAL TEÓRICO}

Esta seção do trabalho trata do referencial teórico relevante para a implementação da pesquisa, enfatizando a intenção de recompra e seus construtos antecedentes, sendo eles o valor percebido, o comprometimento afetivo, o comprometimento normativo e a propaganda boca a boca positiva.

\subsection{CONSTRUTOS ABORDADOS}

O valor percebido desponta como o primeiro construto a ser investigado, devido às rápidas mudanças no ambiente empresarial, onde há um número crescente de consumidores mais exigentes, diante de uma expansão no número de alternativas de fornecedores, marcas, produtos e/ou serviços. Diante disso, o valor percebido é considerado fator chave para a conquista do consumidor em um ambiente altamente competitivo, sendo muito importante para o sucesso das organizações (RUIZ et al., 2008).

Outra reflexão é que o atual ambiente dos negócios, exige que as empresas conheçam profundamente seus clientes, sendo capaz de criar valor para eles e obter a sua preferência ou o seu comprometimento do consumidor em relação à empresa, sua marca ou sua oferta (LEE; EZE; NDUBISI, 2011). Uma série de pesquisadores vem afirmando que o valor percebido pelo consumidor é uma variável antecedente do comprometimento (JOHNSON; HERRMANN; HUBER, 2006; FULLERTON, 2011; THAICHON et al., 2014).

Moliner et al. (2007) sugerem que o comprometimento está relacionado ao mais alto nível de vínculo relacional entre as partes. A essência do comprometimento entre as partes está na criação, desenvolvimento e manutenção de um relacionamento em longo prazo, havendo o desejo, de ambas as partes, fazer sacrifícios de curto e médio prazo, a fim de obter benefícios em uma perspectiva futura (TUMMALA; PHILLIPS; JOHNSON, 2006). Sendo assim, pode-se dizer que quanto maior o grau de dependência entre as partes, maior seria o interesse na sustentação do relacionamento sob uma perspectiva de longo prazo, ou seja, quanto maior o comprometimento entre as partes, maior será a intenção de recompra (MORGAN; HUNT, 1994; PALMATIER et al., 2013).

É importante salientar, ainda, que alguns estudos consideram o comprometimento com uma visão multidimensional. Alguns autores concordam que o comprometimento deve incluir as dimensões afetiva, calculativa e normativa. Em essência, o comprometimento afetivo se refere ao "querer" manter um relacionamento. O comprometimento calculista (ou calculativo) 
instiga as partes a ponderarem sobre o "ter" (possíveis ganhos) para manter o relacionamento. E, por fim, o comprometimento normativo, que diz respeito ao sentimento de como você "deve" agir ou se comportar para manter o relacionamento (GRUEN; SUMMERS; ACITO, 2000). Porém, neste estudo, optou-se testar o comprometimento afetivo e o comportamento normativo pelo fato de se entender que o comprometimento calculativo apresenta menor relevância para o contexto em estudo.

Suportado por Johnson, Herrmann e Huber (2006), o valor percebido apresenta uma associação positiva com o comprometimento afetivo, observando que o valor percebido reflete o que o consumidor ou o cliente deseja do fornecedor. Aliás, Hur, Kim e Kim (2013) verificaram que o valor percebido influencia no surgimento do compromisso afetivo do consumidor ou cliente com a empresa no contexto de serviço de telefonia móvel.

Neste contexto, quando o consumidor, perceber um alto valor de um produto e/ou serviço de uma determinada empresa ou marca poderá fazer com que permaneça com a empresa, aumentando suas motivações ou convicções, criando e ampliando o seu comprometimento afetivo em relação a ela (MUSA; PALLISTER; ROBSON, 2005). Diante disso, segue a formulação da primeira hipótese de pesquisa:

H1: O valor percebido pelo consumidor apresenta um efeito positivo e direto sobre o seu comprometimento afetivo em relação à marca do produto.

De outra forma, o comprometimento normativo infere em sustentar os relacionamentos devido às normas e/ou às regras que são preestabelecidas pelos atores envolvidos (KEININGHAM et al., 2015). Dessa forma, o comprometimento normativo pode ser definido como o grau no qual um comprador está psicologicamente ligado à determinada empresa (ou marca), com base em seu senso de obrigação moral, gerando uma responsabilidade formal (BANSAL; IRVING; TAYLOR, 2004; PARK; RAINEY, 2007). Quando um indivíduo é normativamente comprometido com uma empresa, ele sente que continuar estando envolvido com esta empresa é a coisa certa a fazer e, portanto, tal comportamento deve ser mantido (FULLERTON, 2011).

Em seu estudo, Fullerton (2014) afirma que existe uma relação positiva entre o valor percebido e o comprometimento normativo. Isto sugere que o comprometimento normativo altera a inclinação das intenções do comprador em relação ao produto ou ao serviço em análise, significando que os clientes podem ser menos sensíveis a mudanças no nível de qualidade percebida se a empresa trabalhar com estratégias e ações que constroem o 
comprometimento normativo no âmbito do seu relacionamento com os clientes.

Alguns estudos suportaram a hipótese de que o valor percebido influencia o comprometimento, em sua maioria usando a dimensão do comprometimento afetivo (JOHNSON; HERRMANN; HUBER, 2006; MOLINER et al., 2007). Porém, o comprometimento normativo é pouco estudado, e permanece em evolução, pois poucos estudos examinaram a natureza de seus antecedentes e consequentes (BANSAL; IRVING; TAYLOR, 2004). Para tanto, a segunda hipótese de pesquisa formulada é a de que:

H2: O valor percebido pelo consumidor apresenta um efeito positivo e direto sobre o seu comprometimento normativo em relação à marca do produto.

Outro construto antecedente da intenção de recompra é a propaganda boca a boca positiva, baseado na indicação de Palmatier et al. (2006), os quais salientam que a implantação bem sucedida de um modelo relacional por parte das empresas junto aos seus clientes pode gerar como resultado a recomendação da empresa para outros clientes em potencial, o chamado boca a boca. Sendo assim, pesquisadores e profissionais da área de marketing devem estar cientes de que os consumidores se valem da propaganda boca a boca como uma fonte de informação relevante antes de tomar decisões de compra, mesmo que a influência da propaganda boca a boca seja relevante e perniciosa em julgamentos de qualidade e fortemente influenciadora nas decisões de compra (LEE; CRANAGE, 2014).

Da mesma forma, Gosling e Lago (20066) e Matos e Rossi (2008) sugerem que o valor percebido tem um impacto positivo sobre a possibilidade dos consumidores se envolverem na propagação de uma propaganda boca a boca positiva sobre uma marca, um produto e/ou um serviço. Para Sirdeshmukh, Singh e Sabol (2002), o valor percebido influencia positivamente as predisposições comportamentais dos clientes. Inclusive, potencializando a propaganda boca a boca. Por sua vez, Petrick (2004) identificou que o valor percebido leva o consumidor à predisposição a recompra com a empresa, e que tais intenções geram comentários positivos, ou seja, estimulam a propaganda boca a boca positiva sobre a experiência vivenciada. Diante disso, apresenta-se a terceira hipótese de pesquisa:

H3: O valor percebido pelo consumidor apresenta um efeito positivo e direto sobre a possibilidade de propagar propaganda boca a boca positiva em relação à marca do produto a outros consumidores potenciais.

Além disso, o comprometimento do consumidor em relação à empresa, e sua marca, é 
um componente vital na propagação da propaganda boca a boca positiva (HAN; RYU, 2012). Reforçando esta ideia, Hur, Kim e Kim (2013) mencionam que o cliente que estiver afetivamente comprometido com uma empresa ou marca, tendenciosamente, estão dispostos a se envolver em uma propaganda boca a boca positiva. Outros estudos também encontraram uma relação positiva entre o comprometimento e a propaganda boca a boca positiva (KIM; HAN; LEE, 2001; FULLERTON, 2011).

Em seu estudo, Brown et al. (2005) evidenciaram o principal efeito do comprometimento, em grande parte operacionalizado como comprometimento afetivo, sobre as intenções positivas da propaganda boca a boca dos consumidores. A justificativa para esta descoberta é que quando os consumidores estão afetivamente empenhados por uma empresa, desejam que a empresa tenha sucesso e que outros consumidores compartilhem de tal sentimento (FULLERTON, 2011). Em acréscimo, Fullerton (2011) destaca que os consumidores mais envolvidos em manter um relacionamento com uma empresa, apresentam maior possibilidade de fazer recomendações à terceiros, ou seja, tendem a se envolver na disseminação de propaganda boca a boca positiva. Desse modo, emerge a quarta hipótese de pesquisa:

H4: O comprometimento afetivo do consumidor em relação à marca do produto apresenta um efeito positivo e direto sobre a possibilidade de propagar propaganda boca a boca positiva em relação à marca do produto a outros consumidores potenciais.

Neste horizonte, nota-se que o comprometimento afetivo do consumidor em relação a uma empresa ou uma marca acontece quando o consumidor tem um forte vínculo afetivo com a empresa ou com alguém que lhe atenda, podendo adquirir repetidamente o mesmo produto e/ou serviço. Sendo assim, consumidores que estão comprometidos com uma empresa ou marca podem vir a se tornarem consumidores retidos ou leais, adotando um comportamento de compras repetidas (ERCIS et al., 2012).

Alguns estudos apontam que o comprometimento afetivo apresenta uma associação positiva em relação à intenção de recompra do consumidor, observando-se que o princípio básico intrínseco ao comprometimento afetivo é a intenção do consumidor em continuar próximo à empresa ou à marca escolhida (JONES et al., 2007). Portanto, consumidores que estão comprometidos afetivamente com a empresa ou a sua marca podem vir a se tornarem consumidores retidos e, até mesmo, leais a esta empresa ou esta marca, apresentando intenção ou mesmo um comportamento de repetição de compras ao longo do tempo (ERCIS et al., 
2012), o que repercute positivamente nos resultados da empresa em termos mercadológicos e econômicos (JOHNSON; HERRMANN; HUBBER, 2006; KIM; OK, 2009).

No que se refere ao comportamento do consumidor, o compromisso afetivo reflete o apego emocional do cliente com o produto e/ou serviço, com a marca e a empresa e, por isso, demonstra uma forte relação com a intenção de recompra (KIM; OK, 2009). Assim sendo, é possível apresentar a quinta hipótese de pesquisa:

H5: O comprometimento afetivo do consumidor em relação à marca do produto apresenta um efeito positivo e direto sobre a intenção de recompra de outros produtos da mesma marca.

Os pesquisadores observam a importância de haver uma maior compreensão sobre os antecedentes e os consequentes do compromisso normativo (BANSAL; IRVING; TAYLOR, 2004), pelo fato de ter evidências que poucos estudos abordaram a relação do comprometimento normativo com as atitudes comportamental do cliente ou consumidor (CATER; CATER, 2010). De acordo com Alves, Terres e Santos (2013), o comprometimento normativo é positivamente associado com a intenção de recompra, salientando que os clientes e/ou consumidores que apresentaram uma obrigação moral com a empresa tende exercer sua recompra futuramente.

Vale destacar que o comportamento normativo compreende o compartilhamento de normas, crenças e valores (internos e externos), que são preestabelecidas pelos atores do relacionamento, os quais sustentam as intenções de recompra dos consumidores em relação a uma determinadamarca (KEININGHAM et al., 2015). Considerando as discussões em torno do tema, foi formulada a sexta hipótese de pesquisa:

H6: O comprometimento normativo do consumidor em relação à marca do produto apresenta um efeito positivo e direto sobre a intenção de recompra de outros produtos da mesma marca.

Em acréscimo, pesquisadores relatam que a propaganda boca a boca desempenha um papel importante no processo de seleção de fornecedores ou de prestadores de serviços e na escolha de marcas, produtos e/ou serviços (GILLY et al., 1998). Em complemento a isso, alguns estudos evidenciaram que a propaganda boca a boca é uma fonte primária de influência informacional sobre a tomada de decisão de compra ou de consumo, ou a intenção de recompra, bem como um veículo para expressar a satisfação ou a insatisfação do consumidor com uma experiência relacionada a um produto e/ou serviço (KITAPCI; 
AKDOGAN; DORTYOL, 2014; MATOS; ROSSI, 2008).

Diante disso, Liu e Lee (2016) enfatizam que a propaganda boca a boca positiva pode influenciar os consumidores a adquirirem novamente a mesma marca ou o mesmo produto e/ou serviço. Para tanto, os consumidores que estão dispostos a fazer comunicações (propaganda boca a boca) positivas são mais susceptíveis de permanecerem no relacionamento, pois mantêm ao longo do tempo a sua intenção de recompra de determinadas empresas ou marcas (WANGENHEIM; BAYON, 2004). Nesta direção, pode-se apresentar a sétima hipótese de pesquisa:

H7: A propaganda boca a boca positiva apresenta um efeito positivo e direto sobre a intenção de recompra de outros produtos da mesma marca.

\subsection{MODELO TEÓRICO PROPOSTO E HIPÓTESES DE PESQUISA}

Para facilitar a compreensão, a partir da discussão teórica realizada, na Figura 1 é apresentado graficamente o Modelo Teórico proposto, por meio do qual se propõe investigar a relação entre os construtos valor percebido, comprometimento afetivo, comprometimento normativo e propaganda boca a boca positiva como antecedentes da intenção de recompra dos consumidores no contexto de compras de smartphones de uma determinada marca.

Figura 1 - Modelo teórico proposto

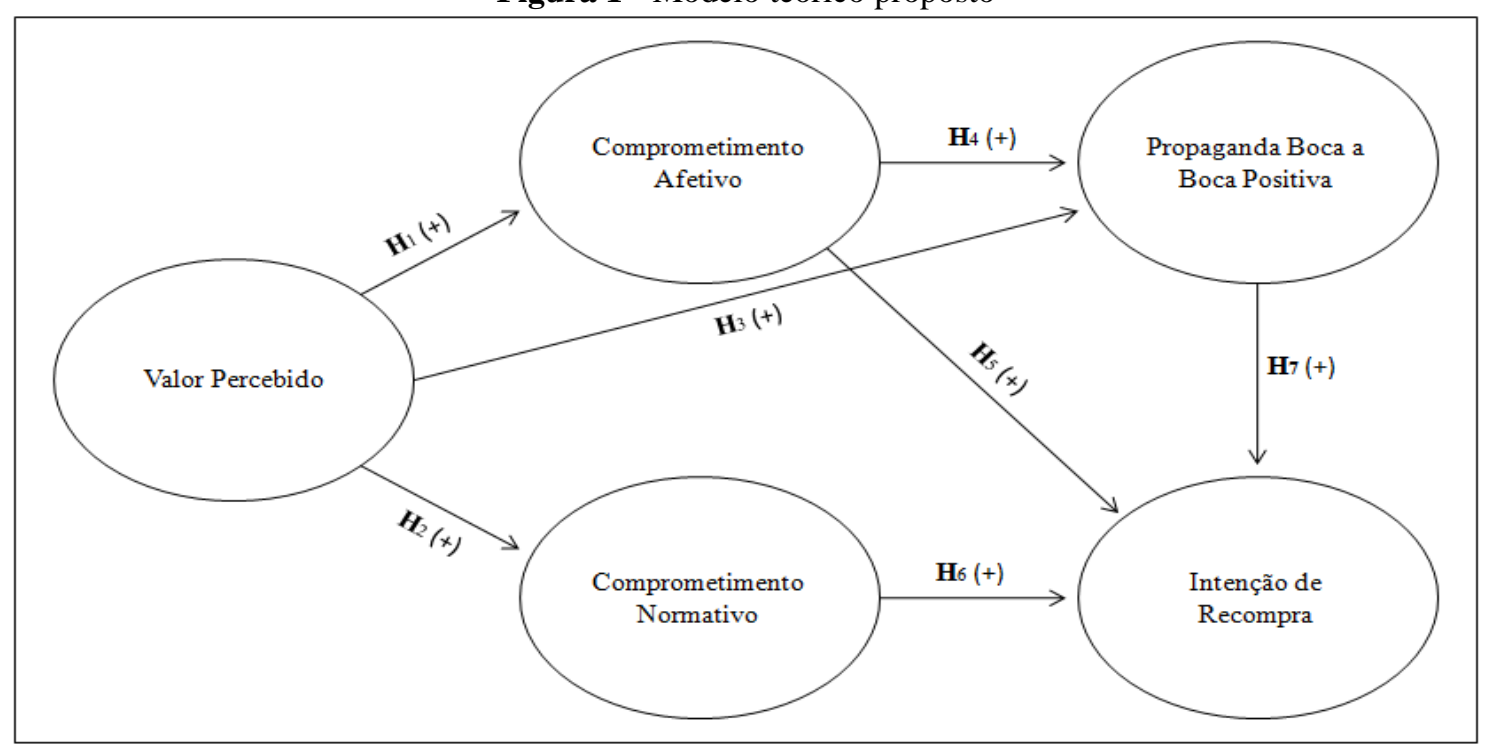

Fonte: elaborada pelos autores com base na literatura (HARRISON-WALKER, 2001; OLARU; PURCHASE; PETERSON, 2008; FULLERTON, 2011; ERCIS et al., 2012; ALVES; TERRES; SANTOS, 2013; KITAPCI; AKDOGAN; DORTYOL, 2014). 


\section{MÉTODO DE PESQUISA}

\subsection{TIPO DE PESQUISA E SUA OPERACIONALIZAÇÃO}

$\mathrm{Na}$ presente pesquisa, foi utilizado o método quantitativo descritivo, por meio da aplicação de uma survey (FINK, 2013), pelo qual o pesquisador passa a obter informações mais exatas, devido às amostras serem amplas e representativas, permitindo verificar as relações entre as variáveis propostas e a validação das relações existentes (HAIR Jr. et al., 2010). Portanto, este método foi aplicado a uma amostra de uma população-alvo, ou seja, compradores da segunda marca de smartphones mais vendida no mundo, com base em um questionário estruturado e com perguntas padronizadas, com a intenção de provocar informações exclusivas dos respondentes sobre seus comportamentos, características, percepções, motivações e intenções (HAIR Jr. et al., 2010; MALHOTRA; BIRKS; WILLS, 2012). Para a análise dos dados foi utilizada a Modelagem de Equações Estruturais, a qual é uma técnica que compõe um conjunto de modelos estatísticos que buscam explicar as relações entre múltiplas variáveis (KLINE, 2011; BYRNE, 2010).

A coleta de dados foi realizada através de um questionário estruturado, que foi entregue pelos próprios pesquisadores para os alunos de uma universidade da Serra Gaúcha. O questionário foi preenchido pelo método do autopreenchimento por parte dos participantes da pesquisa, sem interferência do pesquisador tanto no entendimento quanto nas percepções dos respondentes (HAIR Jr. et al., 2010). Considerando os critérios definidos para a pesquisa, foi determinada uma amostra aleatória, a qual totalizou 444 casos válidos.

Na elaboração do questionário de pesquisa, foi adotada uma escala intervalar do tipo Likert de sete pontos (NETEMEYER; BEARDEN; SHARMA, 2003), tendo em seus extremos "1. Discordo Totalmente" a "7. Concordo Totalmente". Destaca-se que as escalas utilizadas foram operacionalizadas a partir de estudos anteriores, conforme mostra o Quadro 1.

O passo seguinte foi a aplicação de um pré-teste, conforme recomendações da literatura (HAIR Jr. et al., 2010; MALHOTRA; BIRKS; WILLS, 2012), aplicado a vinte respondentes com características (perfil) similares às da população-alvo da pesquisa, ou seja, compradores (alunos) de smartphone da segunda marca mais vendida no mundo. De uma forma geral, os respondentes não tiveram dificuldades na compreensão das questões, sendo que não foi necessário nenhum ajuste no questionário. Cabe ressaltar que tais questionários não foram incorporados à amostra final da pesquisa. 
Quadro 1 - Operacionalização dos construtos

\begin{tabular}{|c|c|c|}
\hline Construtos & Variáveis & Autores \\
\hline Valor Percebido & VALOR_1 a 4 & Perin et al. (2004) e Sirdeshmukh, Singh e Sabol (2002) \\
\hline $\begin{array}{l}\text { Comprometimento } \\
\text { Afetivo }\end{array}$ & COMP AFE_1 a 4 & Jones et al. (2007) e Alves, Terres e Santos (2013) \\
\hline $\begin{array}{l}\text { Comprometimento } \\
\text { Normativo }\end{array}$ & COMP NORM_1 a 4 & $\begin{array}{l}\text { Meyer e Allen (1991), Bansal, Irving e Taylor (2004) e } \\
\text { Alves, Terres e Santos (2013) }\end{array}$ \\
\hline $\begin{array}{l}\text { Propaganda Boca } \\
\text { a Boca Positiva }\end{array}$ & PBP_1 a 4 & Brown et al. (2005) e Alves, Terres e Santos (2013) \\
\hline Intenção de Recompra & INT RECP_1 a 4 & $\begin{array}{l}\text { Jones et al. (2007), Zeithaml, Berry e Parasuraman } \\
\text { (1996), Alves, Terres e Santos (2013) e Teixeira e } \\
\text { Hernandez (2012) }\end{array}$ \\
\hline
\end{tabular}

Obs.: COMP AFE_1 e 2 - itens de escala com escores reversos.

Fonte: elaborado pelos autores.

\subsection{PROCEDIMENTOS DE PREPARAÇÃO E ANÁLISE DOS DADOS}

A etapa de preparação dos dados foi realizada com a intenção de verificar as características e o ajuste dos dados coletados, com a intenção de identificar os dados omissos (missings), bem como as observações atípicas (outliers). Para a verificação dos missings foi utilizada a técnica listwise deletion (BYRNE, 2010), pela qual se considera somente os questionários com dados completos. Assim, doze questionários foram eliminados, resultando dos 444 questionários coletados, 432 casos. A seguir, foram verificados os outliers, utilizando uma combinação de análises uni e multivariadas (HAIR Jr. et al., 2009).

Primeiramente, foi aplicada a verificação dos escores padrão ( $\mathrm{Z}$ scores) univariados, sendo que outros doze casos foram eliminados por apresentaram valores superiores a $|3|$ (HAIR Jr. et al., 2009), reduzindo a amostra para 420 casos. Além disso, foram investigados os outliers multivariados por meio do cálculo da distância de Mahalanobis ( $\left.{ }^{2}\right)$. Considerando uma significância de $\mathrm{p}<0,005$ e os graus de liberdade $(\mathrm{gl}=19)$, indicando mais nove casos para a exclusão, resultando em uma amostra final de 411 casos ( $n=411)$.

O último passo da preparação da análise dos dados foi composto pelos testes das suposições inerentes às bases estatísticas da análise multivariadas. Para tanto, foi verificada a normalidade, a homoscedasticidade, a linearidade e a multicolinearidade (HAIR Jr. et al., 2009; KLINE, 2011; MALHOTRA; BIRKS; WILLS, 2012), sendo que todos os resultados foram satisfatórios por apresentarem valores conforme o recomendado na literatura. 


\section{APRESENTAÇÃO DOS RESULTADOS}

\subsection{CARACTERIZAÇÃO DA AMOSTRA}

Para a caracterização da amostra, foram coletadas informações a respeito do gênero (sexo), do nível de renda mensal, do serviço que o respondente utiliza com maior frequência em seu smartphone e se já haviam comprado ou utilizado outras marcas de smartphone. Analisando-se o sexo dos respondentes, verificou-se que 57,4\% dos entrevistados são do sexo feminino (236 mulheres) e 42,6\% do sexo masculino (175 homens). Em se tratando do nível de renda individual mensal dos respondentes, evidenciaram-se rendas de dois salários mínimos até renda de mais de 20 salários mínimos (considerando-se o salário mínimo de $\mathrm{R} \$ 788,00)$.

Em se tratando do serviço utilizado com maior frequência no smartphone, dos 411 usuários de smartphone da marca em estudo, ou seja, participantes que compuseram a amostra final da pesquisa, 65,5\% deles (ou 269 respondentes) utilizam predominantemente o acesso a redes sociais, seguido de ligações telefônicas com 27,7\% (ou 114 respondentes), para pesquisa 2,9\% (ou 12 respondentes), para jogos 2,4\% (ou 10 respondentes), e outras finalidades com $1,5 \%$ (ou 6 respondentes), conforme mostra a Tabela 1.

Tabela 1 - Distribuição dos serviços utilizados no smartphone

\begin{tabular}{ccc}
\hline Serviço Utilizado com Maior Frequência & Frequência & \% \\
\hline Acesso a Redes Sociais & 269 & 65,5 \\
\hline Ligações Telefônicas & 114 & 27,7 \\
\hline Pesquisa & 12 & 2,9 \\
\hline Jogos & 10 & 2,4 \\
\hline Outras Finalidades & 6 & 1,5 \\
\hline Total & 411 & 100,0 \\
\hline
\end{tabular}

Fonte: elaborada pelos autores.

\subsection{VALIDAÇÃO INDIVIDUAL DOS CONSTRUTOS}

A seguir, deu-se início à validação individual dos construtos, com a intenção de medir o quanto um conjunto de variáveis testadas representa o construto latente (HAIR Jr. et al., 2009). Para a verificação da unidimensionalidade foi implementada a Análise Fatorial Exploratória (AFE), por meio de componentes principais e através da rotação ortogonal 
Varimax (AFIFI; MAY; CLARK, 2012; JOHNSON; WICKERN, 2007), sendo que as cargas fatoriais da AFE variaram entre 0,542 e a 0,861, com um índice de 0,000 no teste de Bartlett e 0,925 no teste Kaiser-Meyer-Olkin (KMO), seguindo a indicação recomendada pela literatura (JOHNSON; WICKERN, 2007; MALHOTRA; BIRKS; WILLS, 2012).

Além disso, foi verificado o Alpha de Cronbach, que apresentou valores entre 0,682 e 0,962; e a confiabilidade composta, obtendo-se valores entre 0,751 e 0,978. Dando sequência à validação dos construtos, foi verificada a validade convergente por meio da Análise Fatorial Confirmatória (AFC), considerando como parâmetros valores acima de 0,5 (KLINE, 2011). Na Tabela 2, portanto, são apresentados os resultados relativos à variância explicada, ao Alpha de Cronbach, à confiabilidade composta e à variância extraída.

Tabela 2 - Variância explicada, Alpha de Cronbach, confiabilidade composta variância extraída

\begin{tabular}{ccccc}
\hline Construtos & $\begin{array}{c}\text { Variância } \\
\text { Explicada }\end{array}$ & $\begin{array}{c}\text { Alpha de } \\
\text { Cronbach }\end{array}$ & $\begin{array}{c}\text { Confiabilidade } \\
\text { Composta }\end{array}$ & $\begin{array}{c}\text { Variância } \\
\text { Extraída }\end{array}$ \\
\hline Valor Percebido & $64,88 \%$ & 0,814 & 0,887 & $66,60 \%$ \\
\hline Comprometimento Afetivo & $79,98 \%$ & 0,682 & 0,751 & $47,30 \%$ \\
\hline Comprometimento Normativo & $67,18 \%$ & 0,835 & 0,900 & $69,60 \%$ \\
\hline Propaganda Boca a Boca Positiva & $89,96 \%$ & 0,962 & 0,978 & $91,90 \%$ \\
\hline Intenção de Recompra & $81,14 \%$ & 0,922 & 0,956 & $84,70 \%$ \\
\hline
\end{tabular}

Em relação à variância extraída, o construto Comprometimento Afetivo apresentou o índice de 0,47, estando em nível um pouco abaixo do recomendado $(0,5)$, porém aceitável, estando na zona de fronteira (HAIR Jr. et al., 2009), enquanto que os demais foram superiores ao recomendado pela literatura.

Por sua vez, a validade discriminante foi testada através do cálculo das variâncias compartilhadas, com base no método proposto por Fornell e Larcker (1981), no qual as variâncias extraídas dos construtos são comparadas com as variâncias compartilhadas. Os resultados apresentaram uma validade discriminante adequada em três construtos em análise, de acordo com a Tabela 3.

Os resultados indicaram que a variância extraída do construto Comprometimento Afetivo $(0,473)$ apresentou-se inferior à variância compartilhada com o construto Propaganda Boca a Boca Positiva $(0,532)$ e o construto Intenção de Recompra $(0,633)$, evidenciando uma possível redundância entre os construtos pelos mesmos estarem fortemente correlacionados. Por outro lado, os resultados confirmam a validade discriminante entre os demais construtos, 
visto que a variância extraída atingiu valores maiores que a variância compartilhada.

Tabela 3 - Validade discriminante

\begin{tabular}{|c|c|c|c|c|c|}
\hline Construtos & $\begin{array}{c}\text { Valor } \\
\text { Percebido }\end{array}$ & $\begin{array}{c}\text { Comprometimento } \\
\text { Afetivo }\end{array}$ & $\begin{array}{c}\text { Comprometimento } \\
\text { Normativo }\end{array}$ & $\begin{array}{c}\text { Propaganda } \\
\text { Boca a Boca } \\
\text { Positiva } \\
\end{array}$ & $\begin{array}{l}\text { Intenção de } \\
\text { Recompra }\end{array}$ \\
\hline Valor Percebido & 0,666 & & & & \\
\hline $\begin{array}{c}\text { Comprometimento } \\
\text { Afetivo }\end{array}$ & 0,443 & 0,473 & & & \\
\hline $\begin{array}{c}\text { Comprometimento } \\
\text { Normativo }\end{array}$ & 0,193 & 0,412 & 0,696 & & \\
\hline $\begin{array}{c}\text { Propaganda Boca } \\
\text { a Boca Positiva }\end{array}$ & 0,480 & 0,532 & 0,268 & 0,919 & \\
\hline $\begin{array}{l}\text { Intenção de } \\
\text { Recompra }\end{array}$ & 0,442 & 0,633 & 0,459 & 0,602 & $\mathbf{0 , 8 4 7}$ \\
\hline
\end{tabular}

Fonte: elaborada pelos autores.

\subsection{VALIDAÇÃO DO MODELO TEÓRICO PROPOSTO}

A validade do modelo estrutural é realizada a partir da qualidade das medidas ou índices de ajustes (HAIR Jr. et al., 2009; BYRNE, 2010; KLINE, 2011). A partir da estimação do modelo híbrido, iniciou-se a validação do Modelo Teórico, por meio da obtenção das medidas de ajustes, as quais foram resultantes do método de estimação da máxima verossimilhança (MLE), que reflete os resultados das análises das matrizes de covariâncias estimadas e observadas (KLINE, 2011), conforme demonstrado na Tabela 4.

Tabela 4 - Medidas de ajustes do Modelo Teórico

\begin{tabular}{cc}
\hline Medidas de Ajustes & Valores Obtidos \\
\hline GFI & 0,899 \\
\hline AGFI & 0,863 \\
\hline RMSEA & 0,069 \\
\hline TLI & 0,942 \\
\hline NFI & 0,930 \\
\hline CFI & \\
\hline & Fonte: elaborada pelos autores.
\end{tabular}

Observando as medidas de ajuste, verifica-se que o resultado do RMSEA $(0,069)$ atende ao valor recomendado pela literatura, ficando entre 0,05 e 0,08 (HAIR Jr. et al., 2009; BYRNE, 2010; KLINE, 2011). Da mesma forma, as medidas TLI $(0,942)$, NFI $(0,930)$ e CFI $(0,952)$ apresentaram valores superiores ao recomendado, ou seja, superiores a 0,900 (HAIR 
Jr. et al., 2009; BYRNE, 2010; KLINE, 2011). Por sua vez, os valores de GFI (0,899) e AGFI $(0,863)$ apresentaram resultados na zona de fronteira, visto que alcançaram valores superiores a 0,800 , sendo que o sugerido pela literatura são valores superiores a 0,90 , embora estes dois indicadores, em muitos casos, resultem abaixo de 0,900 (BAGOZZI; YI, 2012).

Prosseguindo, o próximo passo da análise dos resultados foi a realização do teste de hipóteses (HAIR Jr. et al., 2009; ; MALHOTRA; BIRKS; WILLS, 2012). Na Tabela 5, portanto, são demonstradas as hipóteses, os caminhos estruturais, os coeficientes não padronizados, os erros-padrão, os coeficientes padronizados, os $t$-values e as probabilidades.

Tabela 5 - Teste de hipóteses

\begin{tabular}{|c|c|c|c|c|c|c|c|}
\hline $\mathbf{H i}$ & $\begin{array}{l}\text { Caminhos } \\
\text { Estruturais }\end{array}$ & $\begin{array}{l}\text { Coeficientes Não } \\
\text { Padronizados (b) }\end{array}$ & Erros & $\begin{array}{c}\text { Coeficientes } \\
\text { Padronizados }(\beta)\end{array}$ & $\begin{array}{c}t- \\
\text { values }\end{array}$ & $\mathbf{P}$ & Resultados \\
\hline H1 & $\begin{array}{l}\text { VALOR } \rightarrow \\
\text { COMP_AFE }\end{array}$ & 0,640 & 0,055 & 0,681 & 11,595 & $\mathrm{p}<0,001$ & Suportada \\
\hline $\mathrm{H} 2$ & $\begin{array}{c}\text { VALOR } \rightarrow \\
\text { COMP_NORM }\end{array}$ & 0,296 & 0,064 & 0,461 & 6,196 & $\mathrm{P}<0,001$ & Suportada \\
\hline H3 & $\mathrm{VALOR} \rightarrow \mathrm{PBP}$ & 0,333 & 0,058 & 0,352 & 5,780 & $\mathrm{p}<0,001$ & Suportada \\
\hline $\mathrm{H} 4$ & $\begin{array}{c}\text { COMP_AFE } \rightarrow \\
\text { PBP }\end{array}$ & 0,503 & 0,048 & 0,501 & 7,836 & $\mathrm{P}<0,001$ & Suportada \\
\hline H5 & $\begin{array}{c}\text { COMP_AFE } \rightarrow \\
\text { INT_RECP }\end{array}$ & 0,375 & 0,066 & 0,322 & 4,294 & $\mathrm{P}<0,001$ & Suportada \\
\hline H6 & $\begin{array}{l}\text { COMP_NORM } \\
\rightarrow \text { INT_RECP }\end{array}$ & 0,424 & 0,087 & 0,249 & 4,502 & $\mathrm{P}<0,001$ & Suportada \\
\hline $\mathrm{H} 7$ & $\begin{array}{c}\text { PBP } \rightarrow \\
\text { INT_RECP }\end{array}$ & 0,494 & 0,094 & 0,426 & 7,496 & $\mathrm{p}<0,001$ & Suportada \\
\hline
\end{tabular}

Avaliando os resultados obtidos pelo teste de hipóteses, confirmou-se que as sete hipóteses foram suportadas estatisticamente, ou seja: H1 (o valor percebido pelo consumidor apresenta um efeito positivo e direto sobre o seu comprometimento afetivo em relação à marca do produto, $\beta=0,681, \mathrm{p}<0,001$ ); $\mathbf{H 2}$ (valor percebido pelo consumidor apresenta um efeito positivo e direto sobre o seu comprometimento normativo em relação à marca do produto, $\beta=0,461, \mathrm{p}<0,001$ ); $\mathbf{H 3}$ (valor percebido pelo consumidor apresenta um efeito positivo e direto sobre, a possibilidade de propagar propaganda boca a boca positiva em relação à marca do produto a outros consumidores potenciais, $\beta=0,352, p<0,001$ ); H4 (o comprometimento afetivo do consumidor em relação à marca do produto apresenta um 
efeito positivo e direto sobre a possibilidade de propagar propaganda boca a boca positiva em relação à marca do produto a outros consumidores potenciais, $\beta=0,501, \mathrm{p}<0,001$ ); H5 (o comprometimento afetivo do consumidor em relação à marca do produto apresenta um efeito positivo e direto sobre a intenção de recompra de outros produtos da mesma marca, $\beta=0,322, p<0,001$ ); H6 (o comprometimento normativo do consumidor em relação à marca do produto apresenta um efeito positivo e direto sobre a intenção de recompra de outros produtos da mesma marca, $\beta=0,249, \mathrm{p}<0,001$ ); e $\mathbf{H 7}$ (a propaganda boca a boca positiva apresenta um efeito positivo e direto sobre a intenção de recompra de outros produtos da mesma marca, $\beta=0,426, \mathrm{p}<0,001)$.

Outra forma de se verificar a efetividade do teste de hipóteses é por meio dos coeficientes de determinação $\left(\mathrm{R}^{2}\right)$, com base nas correlações múltiplas ao quadrado de cada variável dependente, ou seja, quanto maior será o poder de explicação da equação da regressão, melhor será a predição da variável dependente (HAIR Jr. et al., 2009). Os coeficientes de determinação $\left(\mathrm{R}^{2}\right)$ do Modelo Teórico são apresentados na Tabela 6.

Tabela 6 - Coeficientes de determinação

\begin{tabular}{lc}
\hline \multicolumn{1}{c}{ Construtos } & Coeficientes de Determinação $\left(\mathbf{R}^{\mathbf{2}}\right)$ \\
\hline Comprometimento Afetivo (COMP_AFE) & 0,464 \\
\hline Comprometimento Normativo (COMP_NORM) & 0,213 \\
\hline Propaganda Boca a Boca (PBP) & 0,611 \\
\hline Intenção de Recompra (INT_RECP) & 0,763 \\
\hline
\end{tabular}

Fonte: elaborada pelos autores.

Ao analisar os coeficientes de determinação $\left(\mathrm{R}^{2}\right)$ encontrados, constatou-se que 76,3\% da variância da Intenção de Recompra é explicada por suas variáveis independentes (HAIR Jr. et al., 2009), sendo elas a Propaganda Boca a Boca Positiva, o Comprometimento Normativo, o Comprometimento Afetivo e o Valor Percebido. Para a Propaganda Boca a Boca Positiva, obteve-se o resultado de que $61,10 \%$ da sua variância é explicada pelo Comprometimento Normativo, pelo Comprometimento Afetivo e pelo Valor Percebido. Já para o Comprometimento Normativo, 21,30\% de sua variância pode ser explicada pelo Comprometimento Afetivo e pelo Valor Percebido; enquanto que 46,40\% do Comprometimento Afetivo é explicado pelo Valor Percebido.

Em relação à Intenção de recompra, constatou-se que 76,30\% de sua variância pode ser explicada pelos antecedentes testados (Propaganda Boca a Boca Positiva, Comprometimento Normativo, Comprometimento Afetivo e Valor Percebido), apresentando 
um alto poder explicativo (HAIR Jr. et al., 2009). Como se percebe, a Intenção de Recompra, para o contexto em estudo (smartphones da segunda marca mais vendida no mundo), apresentou evidências de que possui um alto poder explicativo a partir dos construtos antecedentes (ou determinantes) contemplados no Modelo Teórico Proposto (vide Figura 1).

\section{DISCUSSÃO DOS PRINCIPAIS RESULTADOS}

O ponto de partida do presente estudo foi compreender as relações dos antecedentes da intenção de recompra dos consumidores que têm sido exploradas na literatura do marketing, mais especificamente no contexto de smartphones, evidenciando alguns achados de pesquisa.

Destaca-se, como primeira evidência, a confirmação da existência do efeito positivo e direto entre o Valor Percebido pelo consumidor sobre o seu Comprometimento Afetivo em relação à marca do produto (smartphone) (H1: VALOR $\rightarrow$ COMP_AFE), sendo que esta relação também foi evidenciada em estudos anteriores (JOHNSON; HERRMANN; HUBER, 2006; HUR; KIM; KIM, 2013), sugerindo que as empresas devem concentrar seus esforços para desenvolver maior comprometimento afetivo do consumidor, através do valor percebido, criando uma ligação emocional entre suas marcas e/ou os produtos comercializados e o consumidor.

A segunda contribuição da pesquisa está relacionada à confirmação da relação que aponta um efeito positivo e direto do Valor Percebido pelo consumidor como antecedente do Comprometimento Normativo $(\mathbf{H} 2:$ VALOR $\rightarrow$ COMP_NORM). Em termos teóricoempíricos existem poucas evidências de estudos que relacionam estes dois construtos (FULLERTON, 2014), o comprometimento normativo é um aspecto pouco compreendido no contexto das práticas relacionais (MEYER; PARFYONOVA, 2010). No entanto, tal relação ainda precisa ser testada com maior amplitude para que se construam mais evidências empíricas sobre a relação entre o Valor Percebido e o Comprometimento Normativo.

A terceira evidência se relaciona à confirmação da existência de um efeito positivo e direto do Valor Percebido como antecedente da Propaganda Boca a Boca Positiva (H3: VALOR $\rightarrow$ PBP), confirmando alguns estudos existentes (como, por exemplo, OLARU; PURCHAS; PETERSON, 2008; GOSLING; LAGO, 2006).

Outra contribuição sustentada pelo Modelo Teórico está relacionada à confirmação da quarta hipótese de pesquisa (H4: COMP_AFE $\rightarrow$ PBP), a qual trata da verificação de um efeito positivo e direto do Comprometimento Afetivo como antecedente da Propaganda Boca a Boca Positiva. A relevância desta contribuição é apontada nos estudos de Kim, Han e Lee 
(2001), Fullerton (2011; 2014) e Han e Ryu (2012), os quais destacam que consumidores e/ou clientes com mais desejo de manter um relacionamento com uma empresa ou com uma marca e que apresentam uma forte atitude positiva ou favorável em relação à empresa ou à marca denotam maior probabilidade de recomendar a mesma para outras pessoas (consumidores e/ou clientes potenciais), engajando-se em propaganda boca a boca positiva, repercutindo em recomendação ou indicação de determinada marca, produto e/ou serviço a outros compradores potenciais.

A quinta constatação sustentada pelo modelo teórico é a confirmação da existência de um efeito positivo e direto do Comprometimento Afetivo como antecedente da Intenção de Recompra dos consumidores (H5: COMP_AFE $\rightarrow$ INT_RECP). Neste sentido, nota-se que os clientes que possuem um forte comprometimento afetivo ou emocional com uma empresa ou uma marca podem se tornar clientes retidos e, até mesmo leais, adotando um comportamento de compra repetida (recompra efetiva) (ERCIS et al., 2012). Aponta-se ainda, a confirmação de que há um efeito positivo e direto do Comprometimento Normativo sobre a Intenção de Recompra dos consumidores (H6: COMP_NORM $\rightarrow$ INT_RECP). Tal evidência se caracteriza como uma importante contribuição teórico-empírica, visto que há poucas evidências de estudos que abordaram a relação entre estes dois construtos (CATER; CATER, 2010). Esta relação também é encontrada no estudo de Bansal, Irving e Taylor (2004), os quais salientam que o comprometimento do consumidor é uma força que leva o indivíduo a continuar comprando um produto e/ou serviço de uma mesma empresa ou marca.

Finalizando, destaca-se a confirmação do efeito positivo e direto da Propaganda Boca a Boca Positiva como antecedente da Intenção de Recompra dos consumidores (H7: PBP $\rightarrow$ INT_RECP). Este achado de pesquisa demonstra que a Propaganda Boca a Boca Positiva causa um impacto relevante sobre as intenções comportamentais dos consumidores, no caso, a intenção de recompra sob uma perspectiva futura, mantendo um relacionamento estreito e duradouro com tal empresa, marca e seus produtos e/ou serviços (WANGENHEIM; BAYON, 2004; KITAPCI; AKDOGAN; DORTYOL, 2014).

Por fim, as contribuições teóricas estão centradas no desenvolvimento de um Modelo Teórico, o qual foi estruturado e testado em um cenário atualmente em constante expansão (o mercado de smartphones), a partir da combinação de diferentes construtos identificados como relevantes na literatura da área, representando, assim, uma evolução dos conceitos empiricamente testados, além de despertar a relevância e a necessidade de realização de novos estudos em torno do tema, em situações de compra ou de consumo com suas especificidades. 


\section{CONSIDERAÇÕES FINAIS}

Devido a expansão do mercado dos telefones celulares, mais especificamente dos smartphones, desperta-se o interesse pela intenção de recompra, sinalizando a necessidade de mudanças estratégicas que devem ser direcionadas para compreensão das necessidades ou desejos dos consumidores, para que as organizações que competem entre si consigam desenvolver relacionamentos de longo prazo, gerando uma maior preferência acerca de suas marcas e produtos comercializados e, consequentemente, maior lucratividade e rentabilidade.

Por meio da presente pesquisa, verificou-se que o valor percebido, pressupondo-se uma análise da diferença entre os benefícios recebidos e os sacrifícios realizados (incorridos) na obtenção de um produto e/ou serviço de determinada marca, influencia positivamente o comprometimento afetivo e o comprometimento normativo do consumidor em relação à empresa fornecedora, à sua marca ou produtos e/ou serviços comercializados.

Este resultado contribui para que se proponha uma reflexão às empresas do segmento de smartphones quanto aos sacrifícios que os clientes realizam para ter os seus produtos, como, por exemplo, tempo investido na escolha da marca ou modelo de produto, bem como quanto aos benefícios que os mesmos obtêm desta relação de compra, como, por exemplo, a qualidade do produto (recursos tecnológicos disponíveis e facilidade de uso), desempenho do produto e benefícios afetivos ou emocionais e sociais e o status que determinada marca ou o produto lhe conferem em relação aos grupos sociais que o indivíduo transita ou pertence.

Pode-se apontar como limitação deste estudo, mesmo que se tenha adotado um rigor metodológico no desenvolvimento desta pesquisa, o fato de que há outros estudos que abordam as marcas de smartphones. Também é possível citar como limitações do trabalho a dúvida sobre a existência de outros fatores (ou construtos), que influenciam tanto na intenção quanto no momento de decisão da recompra de uma marca ou produto. Neste sentido, perante as limitações expostas, conclui-se que deve ser encorajada a realização de outras pesquisas teórico-empírico em relação a esta temática.

De toda forma, este estudo aponta fronteiras para o desenvolvimento de pesquisas futuras, a partir da testagem de outros métodos nestes ou em outros construtos antecedentes da intenção de recompra dos consumidores. É oportuno, por exemplo, verificar o comportamento dos construtos antecedentes da intenção de recompra de uma marca, de seus produtos e/ou de seus serviços por parte tanto dos consumidores em contextos do tipo B2C - Business-toConsumer, quanto por parte dos clientes corporativos em contexto do tipo $\mathrm{B} 2 \mathrm{~B}$ - Business-toBusiness, utilizando-se o método empregado nesta pesquisa ou outros métodos, tais como o 
método experimental, replicando o Modelo Teórico proposto ou desenvolvendo ou testando novos modelos que possam trazer evidências mais robustas acerca dos antecedentes da intenção de recompra ou da compra efetiva de marcas, produtos e/ou serviços.

\section{REFERÊNCIAS}

AFIFI, A.; MAY, S.; CLARK, V. A. Practical multivariate analysis. $5^{\text {th }}$ edition. Boca Raton: Taylor \& Francis Group, 2012.

ALVES, D. A.; TERRES, M. S.; SANTOS, C. P. Custos de mudança e seus efeitos no comprometimento, na intenção de recompra e no boca-a-boca em serviços educacionais.

BASE - Revista de Administração e Contabilidade da Unisinos, v. 10, n. 4, p. 355-372, 2013.

BAGOZZI, R. P.; YI, Y. Specification, evaluation, and interpretation of structural equation models. Journal of the Academic Marketing Science, v. 40, p. 8-34, 2012.

BANSAL, H.; IRVING, P. G.; TAYLOR, S. F. A three-component model of customer commitment to service provider. Academy of Marketing Science, v. 32, n. 3, p. 234-250, 2004.

BROWN, T. J.; BARRY, T. E.; DACIN, P. A.; GUNST, R. F. Spreading the word: investigating antecedents of consumers' positive word-of-mouth intentions and behaviors in a retailing context. Journal of the Academy of Marketing Science, v. 33, n. 2, p. 123-138, 2005.

BYRNE, B. M. Structural equation modeling with AMOS: basic concepts applications, and programming. $2^{\text {nd }}$ edition. New York: Routledge, 2010.

CATER, T.; CATER, B. Product and relationship quality influence on customer commitment and loyalty in B2B manufacturing relationships. Industrial Marketing Management, v. 39, n. 8 , p. 1.321-1.333, 2010.

DINH, T. D.; MAI, K. N. Guerrilla marketing's effects on gen Y's word-of-mouth intention a mediation of credibility. Asia Pacific Journal of Marketing, v. 28, n. 1, p. 4-22, 2016.

ENNEW, C. T.; BANERJEE, A. K.; LI, D. Managing word of mouth communication: empirical evidence from India. International Journal of Bank Marketing, v. 18, n. 2, p. 75$83,2000$.

ERCIS, A.; ÜNAL, S.; CANDAN, F. B.; YILDIRIM, H. The effect of brand satisfaction, trust and brand commitment on loyalty and repurchase intentions. Social and Behavioral 
sciences, v. 58, p. 1395-1404, 2012.

FINK, A. How to conduct surveys: a step-by-step guide. $5^{\text {th }}$ edition. Thousand Oaks: Sage Publications, 2013.

FORNELL, C.; LARCKER, D. Evaluating structural equation models with unobserved variables and measurement error. Journal of Marketing Research, v. 18, n. 1, p. 39-50, 1981.

FULLERTON, G. Creating advocates: The roles of satisfaction, trust and commitment.

Journal of Retailing and Consumer Services, v. 18, n. 1, p. 92-100, 2011.

FULLERTON, G. The moderating effect of normative commitment on the service qualitycustomer retention relationship. European Journal of Marketing, v. 48, n. 3/4 p. 657-673, 2014.

GILLY, M. C.; GRAHAM, J. L.; WOLFINBARGER, M. F.; YALE, L. J. A dyadic study of international information search. Journal of The Academy of Market Science, v. 26, n. 2, p. 83-100, 1998.

GOSLING, M.; LAGO, J. Dimensões do valor percebido e a influência da boca a boca: reflexos teóricos e proposições de um modelo. READ - Revista Eletrônica de Administração, v.12, n. 5, p. 345-368, 2006.

GRUEN, T. W.; SUMMERS, J. O.; ACITO, F. Relationship marketing activities, commitment and membership behaviors in professional associations. Journal of Marketing, v. 64, n. 3, p. 34-49, 2000.

HAIR Jr., J. F.; BLACK, W. C.; BABIN, B. J.; ANDERSON, R. E.; TATHAM, R. L. Análise multivariada de dados. 6. ed. Porto Alegre: Bookman, 2009.

HAIR Jr., J. F.; WOLFINBARGER, M.; ORTINAU, D. J.; BUSH, R. P. Fundamentos de pesquisa de marketing. Porto Alegre: Bookman, 2010.

HAN, H.; KIM, Y. An investigation of green hotel customers' decision formation: developing an extended model of the theory of planned behavior. International Journal of Hospitality Management, v. 29, n. 4, p. 659-668, 2010.

HAN, H.; RYU, K. Key factors driving customers' word-of-mouth intentions in full-service restaurants: the moderating role of switching costs. Cornell Hospitality Quarterly, v. 53, n. 2, p. 103-109, 2012.

HARRISON-WALKER, J. The measurement of word-of mouth communication and an investigation of service quality and customer commitment as potential antecedents. Journal of Service Research, v. 4, n. 1, p. 60-75, 2001.

HE, Y.; SONG, H. A mediation model of tourists' repurchase intentions for packaged tour 
services. Journal of Travel Research, v. 47, n. 3, p. 317-331, 2009.

HELLIER, P. K.; GEURSEN, G. M.; CARR, R. A.; RICKARD, J. A. Customer repurchase intention: a general structural equation model. European Journal of Marketing, v. 37, n. 11, p. 1.762-1.800, 2003.

HSU, M. H.; CHANG, C. M.; CHU, K. K.; LEE, Y. J. Determinants of repurchase intention in online group-buying: the perspectives of DeLone \& McLean is success model and trust.

Computers in Human Behavior, v. 36, p. 234-245, 2014.

HUR, W. M.; KIM, K. H.; KIM, H. Investigation of the relationship between service values and loyalty behaviors under high commitment. Service Business, v. 7, n. 1, p. 103-119, 2013. ISAID, E. M.; FAISAL, M. N. Consumer's repurchase intention towards mobile phone brand in Qatar: an exploratory action framework. Global Business Review, v. 16, n. 4, p. 594-608, 2015.

JOHNSON, M.; HERRMANN, D. A.; HUBER, F. The evolution of loyalty intentions. Journal of Marketing, v. 70, n. 2, p. 122-132, 2006.

JOHNSON, R. A.; WICKERN, D. W. Applied multivariate statistical analysis. $6^{\text {th }}$ edition. Upper Saddle River: Pearson / Prentice Hall, 2007.

JONES, M. A.; DAVID, L.; MOTHERSBAUGH, D. L.; BEATTY, S. E. The positive and negative effects of switching costs on relational outcomes. Journal of Service Research, v. 9, n. 4, p. 335-355, 2007.

KEININGHAM, T. L.; FRENNEA, C. M.; AKSOY, L.; BUOYE, A.; MITTAL, V. A five component customer commitment model: implications for repurchase intentions in goods and services industries. Journal of Service Research, v. 18, n. 4, p. 433-450, 2015.

KIM, W. G.; HAN, J. S.; LEE, E. Effects of relationship marketing on repeat purchase and word of mouth. Journal of Hospitality and Tourism Research, v. 25, n. 3, p. 272-88, 2001. KIM, W.; OK, C. The effects of relational benefits on customers perception of favorable inequity, affective commitment, and repurchase intention in full. Journal of Hospitality \& Tourism Research, v. 33, n. 2, p. 227-244, 2009.

KITAPCI, O.; AKDOGAN, C.; DORTYOL, I. T. The impact of service quality dimensions on patient satisfaction, repurchase Intentions and word-of-mouth communication in the public healthcare industry. Social and Behavioral Sciences, v. 148, n. 2, p. 161-169, 2014.

KLINE, R. B. Principles and practice of structural equation modeling. $3^{\text {rd }}$ edition. New York: The Guilford Press, 2011.

LEE, C. H; CRANAGE, D. A. Toward understanding consumer processing of negative online word-of-mouth communication: the roles of opinion consensus and organizational response 
strategies. Journal of Hospitality \& Tourism Research, v. 38, n. 3, p. 330-360, 2014. LEE, C. H.; EZE, U. C.; NDUBISI, N. O. Analyzing key determinants of online repurchase intentions. Asia Pacific Journal of Marketing and Logistics, v. 23, n. 2, p. 200-221, 2011. LI, H.; HONG, J. Factors influencing consumers' online repurchasing behavior: a review and research agenda. Journal International Business, v. 5, n. 4, p. 161-166, 2013. LIN, C.; LEKHAWIPAT, W. Factors affecting online repurchase intention. Industrial Management \& Data Systems, v. 114, n. 4, p. 597-611, 2014.

LIU, C. H. S.; LEE, T. Service quality and price perception of service: influence on word-ofmouth and revisit intention. Journal of Air Transport Management, v. 52, n. 5, p. 42-54, 2016.

MALHOTRA, N. K.; BIRKS, D.; WILLS, P. Marketing research: applied approach. $4^{\text {th }}$ edition. New York: Pearson, 2012.

MATOS, C. A.; ROSSI, C. A. V. Word-of-mouth communications in marketing: a metaanalytic review of the antecedents and moderators. Journal of the Academic Marketing Science, v. 36, n. 4, p. 578-596, 2008.

MEYER, J. P.; ALLEN, N. J. A three-component conceptualization of organizational commitment. Human Resource Management Review, v. 1, n. 1, p. 61-89, 1991.

MEYER, J. P.; PARFYONOVA, N. Normative commitment in the workplace: a theoretical analysis and re-conceptualization. Human Resource Management Review, v. 20, n. 4, p. 283-294, 2010.

MOLINER, M. A.; SÁNCHEZ, J.; RODRÍGUEZ, R. M.; CALLARISA, L. Perceived relationship quality and post-purchase perceived value: an integrative framework. European Journal of Marketing, v. 41, n. 11/12, p. 1.392-1.422, 2007.

MORGAN, R. M.; HUNT, S. D. The commitment-trust theory of relationship marketing. Journal of Marketing, v. 58, n. 3, p. 20-38, 1994.

MUSA, R.; PALLISTER, J.; ROBSON, M. The roles of perceived value, perceived equity and relational commitment in a disconfirmation paradigm framework: an initial assessment in a 'relationship-rich' consumption environment. Advances in Consumer Research, v. 32, n. 1, p. 349-357, 2005.

NETEMEYER, R. G.; BEARDEN, W. O.; SHARMA, S. Scaling procedures: issues and applications. Thousand Oaks: Sage Publications, 2003.

OLARU, D.; PURCHASE, S.; PETERSON, N. From customer value to repurchase intentions and recommendations, Journal of Business \& Industrial Marketing, v. 23, n. 8, p. 554-565, 2008. 
PALMATIER, R. W.; DANT, R. P.; GREWAL, D.; EVANS, K. R. Factors influencing the effectiveness of relationship marketing: a meta-analysis. Journal of Marketing, v. 70, n. 3, p. 136-153, 2006.

PALMATIER, R. W.; HOUSTON, M. B.; DANT, R. P.; GREWAL, D. Relationship velocity: toward a theory of relationship dynamics. Journal of Marketing, v. 77, n. 1, p. 1330, 2013.

PARK, S. M.; RAINEY, H. G. Antecedents, mediators and consequences of affective, normative and continuance commitment: empirical test of commitment effect in Federal Agencies. Review of Public Personnel Administration, v. 27, n. 3, p. 197-226, 2007. PERIN, M. G.; SAMPAIO, C. H.; BREI, V. A.; PORTO, C. de A. As relações entre confiança, valor e lealdade: um estudo intersetorial. In: XXVIII ENANPAD - Encontro Anual da Associação Nacional dos Programas de Pós-Graduação em Administração. Curitiba: Anais... Curitiba: ANPAD, 2004.

PETRICK, J. F. Development of a multi-dimensional scale for measuring the perceived value of a service. Journal of Leisure Research, v. 34, n. 2, p. 119-134, 2002.

PRITCHARD, M. P.; HAVITZ, M. E.; HOWARD, D. R. Analyzing the commitment-loyalty link in service relationships. Journal of the Academy of Marketing Science, v. 27, n. 3, p. 333-348, 1999.

REVELS, J.; TOJIB, D.; TSARENKO, Y. Understanding consumer intention to use mobile services. Australasian Marketing Journal, v. 18, n. 2, p. 74-80, 2010.

RUIZ, D. M.; GREMLER, D. D.; WASHBURN, J. H.; CARRIÓN, G. C. Service value revisited: specifying a higher-order, formative measure. Journal of Business Research, v. 61, n. 12, p. 78-91, 2008.

SHIN, D. H.; SHIN, Y. J.; CHOO, H.; BEOM, K. Smartphones as smart pedagogical tools: implications for smartphones as u-learning devices. Computers in Human Behavior, v. 27, n. 6, p. 2.207-2.214, 2011.

SIRDESHMUKH, D.; SINGH, J.; SABOL, B. Consumer trust, value, and loyalty in relational exchanges. Journal of Marketing, v. 66, n. 1, p. 15-37, 2002.

TEIXEIRA, J. M.; HERNANDEZ, J. M. da C. Valores de compra hedônico e utilitário: os antecedentes e as relações com os resultados do varejo. Revista Eletrônica de Administração, v. 71, n. 1, p. 130-160, 2012.

THAICHON, P.; LOBO, A. PRENTICE, C.; QUACH, T. N. The development of service quality dimensions for internet service providers: retaining customers of different usage patterns. Journal of Retailing and Consumer Services, v. 21, n. 6, p. 1.047-1.058, 2014. 
TING, D. H.; LIM, S. F.; PATANMACIA, T. S.; LOW, C. G.; KER, G. C. Dependency on smartphone and the impact on purchase behavior. Journal Young Consumers, v. 12, n. 3, p. 193-203, 2011.

TSAI, H. T.; HUANG, H. C. Determinants of e-repurchase intentions: an integrative model of quadruple retention drivers. Information \& Management, v. 44, n. 3, p. 231-239, 2007. TUMMALA, V. M. R.; PHILLIPS, C. L. M.; JOHNSON, M. Assessing supply chain management success factors: a case study. Supply Chain Management, v. 11, n. 2, p. 179192, 2006.

VAERENBERGH, Y. V.; LARIVIÈRE, B.; VERMEIR, I. The impact of process recovery communication on customer satisfaction, repurchase intentions, and word-of-mouth intentions. Journal of Service Research, v. 15, n. 3, p. 262-279, 2012.

WANG, D.; XIANG, Z.; FESENMAIER, D. R. Adapting to the mobile world: a model of smartphone use. Annals of Tourism Research, v. 48, p. 11-26, 2014.

WANGENHEIM, F. V.; BAYON, T. The effect of word-of-mouth on services switching: measurement and moderating variables. European Journal of Marketing, v. 38, n. 9/10, p. 1.173-1.185, 2004.

WU, L. Y.; CHEN, K. Y.; CHEN, P. Y.; CHENG, S. L. Perceived value, transaction cost, and repurchase-intention in online shopping: a relational exchange perspective. Journal of Business Research, v. 67, n. 1, p. 2.768-2.776, 2012.

ZEITHAML, V. A.; BERRY, L. L.; PARASURAMAN, A. The behavioral consequences of service quality. Journal of Marketing, v. 60, n. 2, p. 31-46, 1996. 